\title{
CFD Simulation of Transonic Flow in High-Voltage Circuit Breaker
}

\author{
Xiangyang Ye and Mahesh Dhotre \\ Interrupter Development, ABB Switzerland Ltd, 5401 Baden, Switzerland \\ Correspondence should be addressed to Xiangyang Ye, xiangyang.ye@ch.abb.com
}

Received 25 October 2011; Revised 23 January 2012; Accepted 8 February 2012

Academic Editor: Nandkishor Nere

Copyright ( $) 2012$ X. Ye and M. Dhotre. This is an open access article distributed under the Creative Commons Attribution License, which permits unrestricted use, distribution, and reproduction in any medium, provided the original work is properly cited.

\begin{abstract}
A high-voltage circuit breaker is an indispensable piece of equipment in the electric transmission and distribution systems. Transonic flow typically occurs inside breaking chamber during the current interruption, which determines the insulating characteristics of gas. Therefore, accurate compressible flow simulations are required to improve the prediction of the breakdown voltages in various test duties of high-voltage circuit breakers. In this work, investigation of the impact of the solvers on the prediction capability of the breakdown voltages in capacitive switching is presented. For this purpose, a number of compressible nozzle flow validation cases have been presented. The investigation is then further extended for a real high-voltage circuit breaker geometry. The correlation between the flow prediction accuracy and the breakdown voltage prediction capability is identified.
\end{abstract}

\section{Introduction}

High-voltage gas circuit breakers are one of the most important and complex components in the electric transmission and distribution systems, with main function of opening the circuit under fault conditions and interrupt currents from very small values up to the maximum rated short-circuit current. The current interruption process in such device is quite complex and involves interaction of electric arc, gas flow, ablation, and radiation.

In typical self-blast high-voltage circuit breaker (schematic is shown in Figure 1), the plasma arc is ignited after arcing contacts $(1,2)$ separate from each other and breaker uses arc energy to create pressure inside a chamber (3) and to blow the arc, at current zero crossing of the alternating current (ac), inside a PTFE nozzle (4). The clearing of hot gas, after arcing contact (2) leaves the nozzle (4) strongly depends on flow pattern inside the nozzle-diffuser system (4-5) which is complex and contains the formation and location of supersonic flow regions, shock fronts, and flow separation and reattachment regions. Design of such system is important for current interruption and subsequent dielectric recovery (better hot gas clearing), as the flow varies strongly throughout the nozzle diffuser system and its interaction with the electric field generates critical regions characterized by low density and high electric field. The probability of electrical breakdown is higher in such regions.

In capacitive switching, the current interruption occurs at high voltages but low current (no-arc), represents one of the important tests that the breaker needs to pass. During the diffuser design process refered to capactive switching, since the electrical field distribution does not change much with different diffuser shapes, the dielectric performance of the gas can be evaluated only from the fluid dynamic characteristics. That is, for given electric field distribution, it is desirable to attain highest possible flow density in the diffuser (while not blocking the gas flow from the nozzle, which would eventually lead to the failure in thermal interruption), for better dielectric capability. Since the local flow property determines the dielectric strength of insulating gas, it is important to understand the dynamics of the transonic gas flow, in the design and development of highvoltage circuit breakers.

Computational Fluid Dynamics (CFD) offers a great advantage for simulating and visualizing the processes in high-voltage circuit breakers. The application of CFD simulation can save a number of expensive tests and significantly 


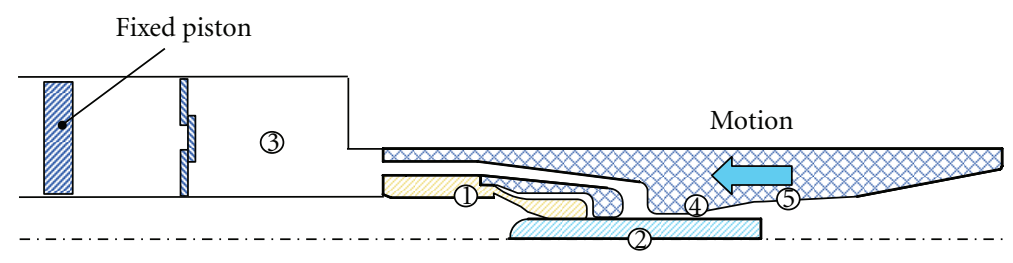

FIGURE 1: Schematic representation of self-blast circuit breaker geometry.

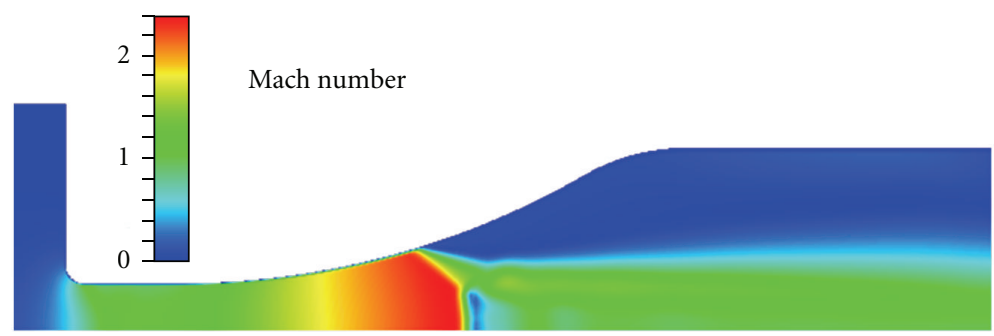

(a)

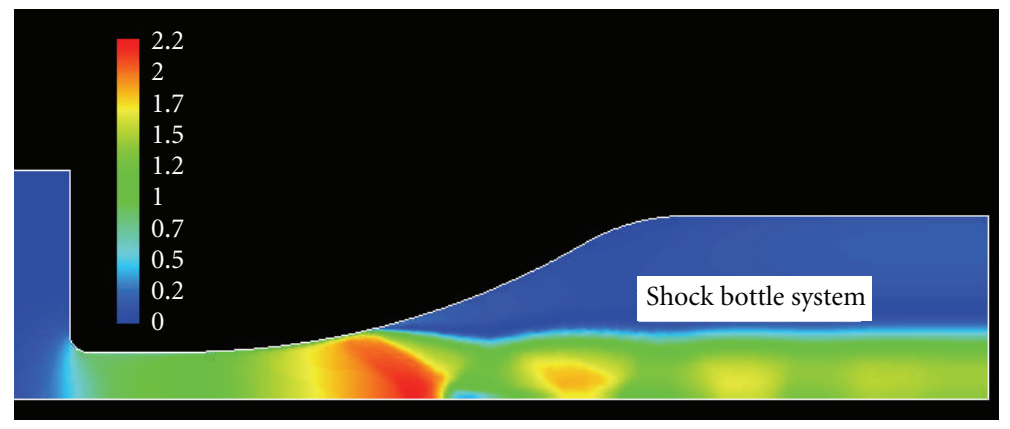

(b)

FIgURE 2: Transonic nozzle simulations for typical diffuser geometries for (a) non-conservative method and (b) conservative method.

accelerate the prototyping, which is directly connected to reduction in cost of breaker development. Since flow in breaker is highly complex and nonlinear transonic compressible flow which allows discontinuous shock structure and complicated flow detachments from the diffuser, a high degree of accuracy of flow calculation is required.

In the present work, a benchmark validation study of two solvers for predicting the breakdown voltages in capacitive switching tests in high-voltage circuit breaker geometry is presented. The semi-implicit pressure correction (SIMPLEC) method [1], which is widely adopted in many commercial CFD tools, and the density-based Jameson scheme with explicit Runge-Kutta time marching method [2] are compared. The in-house code has been used for all simulations and two-dimensional axisymmetric simulations are carried out for performance comparison of the two algorithms.

The paper is organized as follows: the main issues in compressible flow simulations are presented in Section 2. Further, the role of the turbulence modeling for transonic nozzle flow simulations, by presenting a validation case based on a 2D nozzle, is demonstrated. In Section 3, CFD validation study for the transient transonic flow in a simple diffuser geometry with different position of electrode (In Figure 1 contact (2)) is presented. In Section 4, validation study of a compressible flow field with the measured transient flow data from a real high-voltage circuit breaker capacitive switching test duty is performed. In Section 5, influence of the different compressible flow solvers on the prediction of dielectric breakdown voltages in capacitive switching tests is presented. In Section 6, final conclusions are drawn.

\section{Main Concerns in Transonic Flow Simulations}

There are several features that the ideal CFD simulation tool must possess for the high-voltage circuit breaker application. Since the mechanical or arc-induced pressure build-up in the heating volume generates transonic flow in the diffuser area, the CFD simulation must be able to capture the key physical features of the transonic nozzle flow. These are characterized by the qualities of capturing exact shock location, shockinduced flow separation from the diffuser wall, and the extension of the recirculation zone; see for illustration Figure 2.

The CFD solves the algebraic difference equations which are derived from the partial differential equations, using different space and time discretization methods. One of the widely used methods is to solve a locally elliptic equation for 
$p$ and determines other primitive flow variables iteratively. This pressure-correction-based algorithm is widely used in many of the commercial tools. On the other hand, one can make use of the hyperbolic characteristics of the NavierStokes equations. Since the flow speed cannot exceed the sonic velocity relative to the background flow velocity, the explicit time marching based on CFL condition [3] can be used. In this case, the solver is based on conserved variables $\vec{U}$ which has an advantage in implementing conservation law constraints on the numerical level.

Conservative and Nonconservative Methodologies. The conservative CFD methods solve the Navier-Stokes equation for conservative variables, the gas density $\rho$, the flow momentum density $\rho \vec{v}$, and the total energy density $E$ :

$$
\begin{gathered}
\frac{\partial}{\partial t} \vec{U}+\nabla \cdot \vec{F}(\vec{U})=\vec{S}, \\
\vec{U} \equiv\left(\begin{array}{c}
\rho \\
\rho \vec{v} \\
E
\end{array}\right), \quad \vec{F}(\vec{U}) \equiv\left(\begin{array}{c}
\rho \vec{v} \\
\rho \vec{v} \otimes \vec{v}+p \mathrm{I} \\
(E+p) \vec{v}
\end{array}\right) .
\end{gathered}
$$

Here, $\vec{v}$ is the flow velocity vector, $p$ is the static pressure, and the vector $\vec{S}$ represents the viscous stress and heat conduction per unit volume. The system of the equation is closed by equation of state which is given by $p=\rho R T$ for thermally ideal gas, where $T$ is temperature in Kelvin and $R$ is the specific gas constant. For a calorically ideal gas, the internal energy $e \equiv(E / \rho)-\left(\vec{v}^{2} / 2\right)$ is given by $e=p /[\rho(y-1)]$, where $y$ is the adiabatic exponent. On the contrary, the non-conservative numerical scheme solve the Navier-Stokes equation for nonconservative primitive variables, $\rho$, $\vec{v}$, and the enthalpy $h$.

In order to compare these methods, simulations are presented in Figure 2 for simple diffuser geometry with inlet-to-outlet pressure ratio typically occurring in the highvoltage circuit breaker. Figure 2 illustrates the difference in shock capturing capabality of non-conservative and conservative methods. Note that the simulation based on nonconservative method does not capture the shock bottle system after the flow detachment, whereas it is clearly captured by the one based on conservative method.

Turbulence Modeling. The prediction capability of the shock induced flow separation and the trailing recirculation zone is dependent on the detailed modeling of the shock and the boundary layer interaction. In order to investigate the effect of turbulence modeling, simulations are carried out for "Sajben diffuser," flow experiments [4]. The tested 2D nozzle in these experiments consists of converging and then diverging nozzle (Figure 3, details about experimental setup is given in [4]). This realizes the supersonic flow which is terminated by a vertical shock located in the downstream area of the throat, for sufficiently high inlet pressures. The vertical shock induces a flow separation with the trailing recirculation zone. The vertical shock position depends on the ratio of the inlet-to-outlet pressures, and the so-called "strong shock" and the "weak shock" cases are examined in

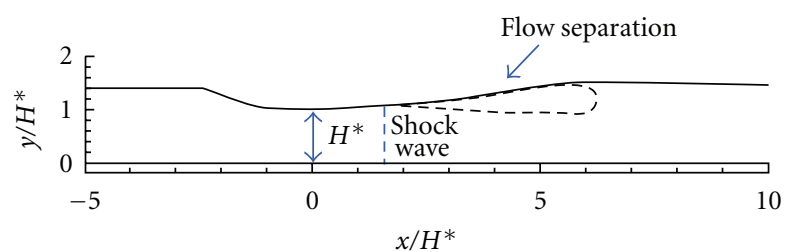

FIGURE 3: The transonic nozzle flow configuration which consists of vertical shock, shock-induced flow separation, and recirculation zone (Sajben Diffuser test case).

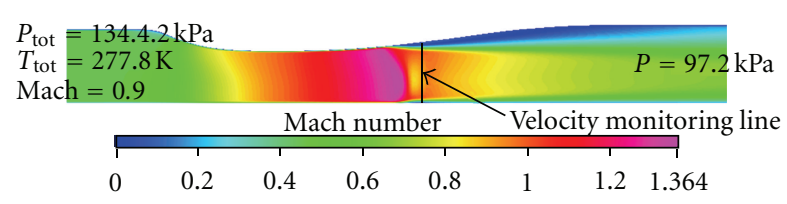

Figure 4: The strong shock flow configuration in Sajben nozzle.

the literature. Here, the steady state simulations are only for the strong shock case.

The two turbulence models have been tested, the widely used $k-\varepsilon$ and RNG $k-\varepsilon$. The size of computation gird used for simulation is in range of $0.1-0.5 \mathrm{~mm}$ and has been selected after careful grid sensitivity study. The strong shock case is characterized by the steady inlet-to-outlet pressure ratio given by $p_{\text {exit }} / p_{0 \text {;inlet }}=0.72$, and the compressible flow features are more dominant than the weak shock case. For the strong shock case, measurements show that the shock is located at approximately 2.5 throat heights into the downstream region of the throat (Figure 4). Figure 5(a) shows the simulated and measured pressure distributions along the top wall. It can be observed that there is a difference in predicted shock locations between the two turbulence models. The RNG $k-\varepsilon$ model captures the shock better compared to $k-\varepsilon$ model. Figure 5(b) shows the simulated and measured velocity profiles along a vertical line located slightly in the downstream region of the shock. Again, the RNG $k-\varepsilon$-based simulation predicts the velocity profile better. As the $k-\varepsilon$-model predicts the shock position further in the downstream region than measurement, the velocity at monitoring line is predicted to be in the upstream region of the shock. This results in the supersonic velocity profile, whereas the RNG $k-\varepsilon$-based simulation predicts subsonic flow along the monitor line. Also, note from Figure 5 that the negative velocities are measured and to a certain extent also better predicted by RNG $k-\varepsilon$-based simulation. This is due to the presence of recirculation region after the flow separation.

\section{Flow Simulation in Presence of a Plug}

The transient transonic flow in a typical diffuser geometry (Figure 6) is often adopted for high-voltage circuit breakers, in the presence of a plug. The measurements are available at given locations and for different plug positions and can be used for good CFD validation; details of measurements are 


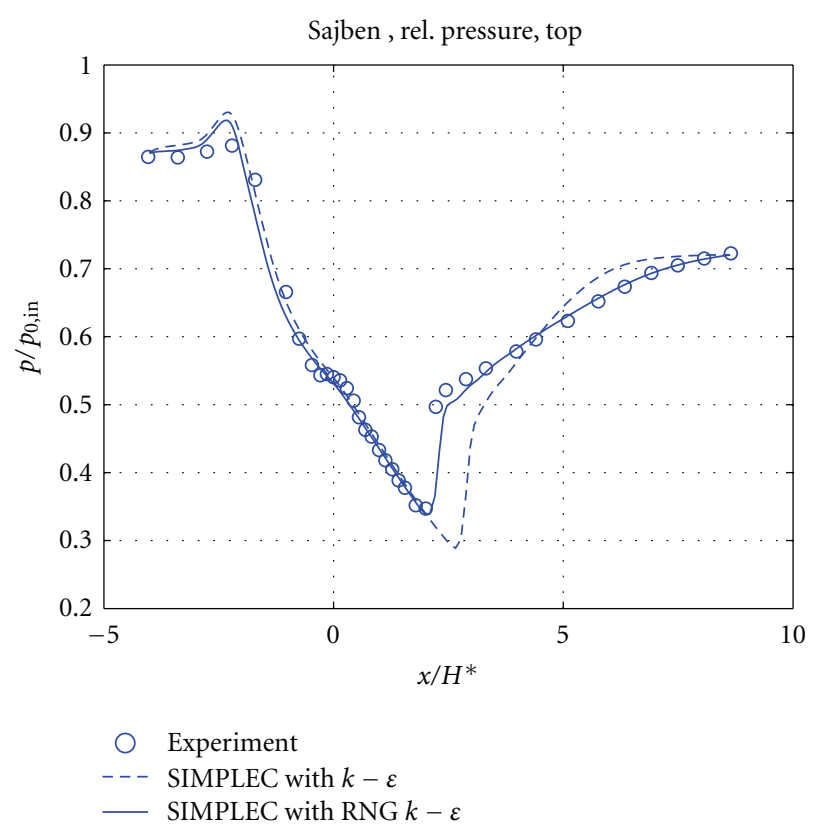

(a)

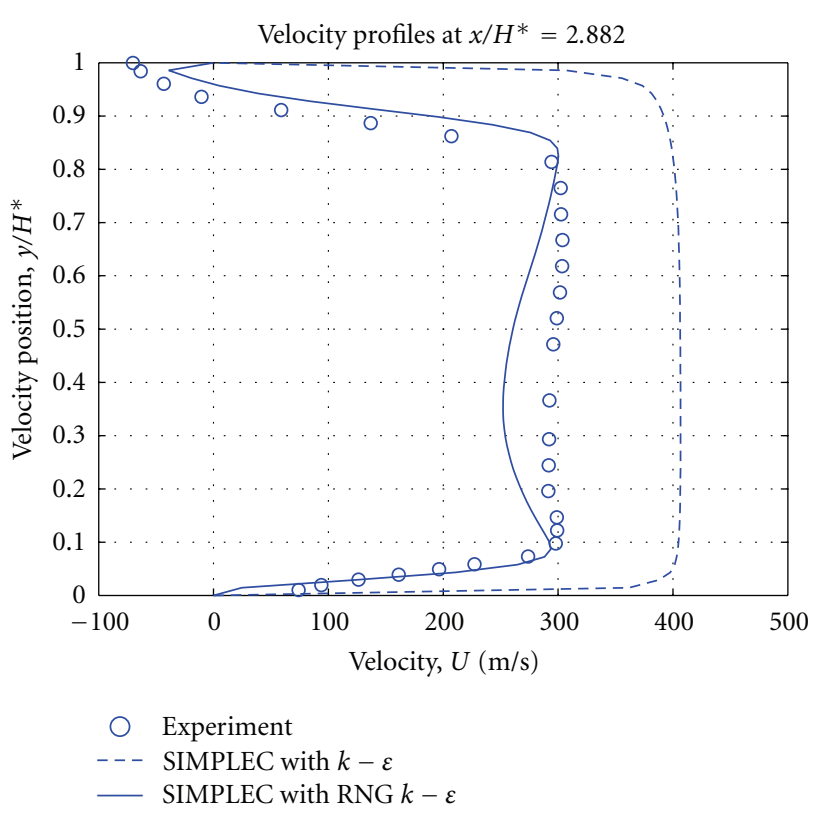

(b)

Figure 5: Simulated and measured (a) pressure profile along the top wall of Sajben nozzle, (b) velocity profiles along the vertical monitoring line at a slightly downstream of the vertical shock.

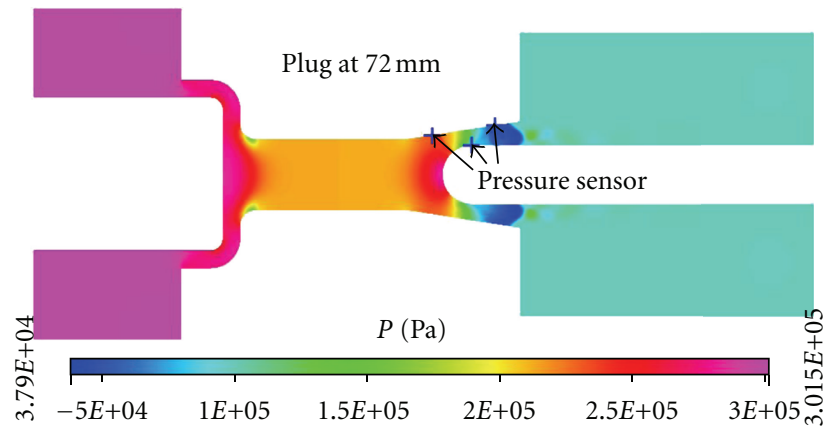

FIgURE 6: Transonic flow configuration at nozzle opening $(72 \mathrm{~mm}$ case).

given in [5]. For the measurements, three pressure sensors were installed, two along the fixed wall in the diffuser region and the one on the plug. The chosen plug positions in experiment, respectively, represent the plug in the main nozzle area (the $42 \mathrm{~mm}$ case), at the nozzle opening (the $72 \mathrm{~mm}$ case), and at the fully open position (the $92 \mathrm{~mm}$ case) during the high-voltage circuit breaker operation.

The transient CFD simulations for the rising inlet pressure from 1 to 3 bars in $25 \mathrm{~ms}$ are performed. The compared benchmark flow simulation algorithms are the SIMPLECbased solver and the Jameson-method-based solver with $k-\varepsilon$ turbulence model. Note that the former is a pressurecorrection-based non-conservative solver and the latter is based on conservative method.
Among the three different plug positions, the plug at the nozzle opening (the " $72 \mathrm{~mm}$ " case) provides the most challenging case for the solver quality evaluations, in terms of capturing the shock. Figure 7 shows the simulated and measured pressure at three different sensor locations. It can be observed that the Jameson-method-based solver with explicit time marching shows better agreement with the measured data. There are two reasons for this. First, because the Jameson method is a conservative scheme, which in principle should have better shock capturing capability, than the non-conservative-method-based SIMPLEC scheme. Second, the Jameson method is based on explicit time marching where the time step size is limited by the sonic speed which transmits local flow information. This explicit scheme should predict better dynamic transient flow phenomena than the SIMPLEC scheme which is based on implicit method.

It can be observed from Figure 7(b) that the SIMPLECscheme-based solver overestimates the supersonic flow expansion along the diffuser than real and predict the shock located farther in the downstream region. Of particular interest is the pressure on the plug (Figure 7(c)). The SIMPLECscheme-based solver overestimates the compressibility on the plug for lower inlet pressure.

\section{Real Circuit Breaker Simulation}

In this section, the comparison of two numerical algorithms (the pressure-correction-based semi-implicit SIMPLEC scheme and the density-based explicit Jameson's method), in predicting transonic flow field configuration inside the high-voltage circuit breaker, is presented. The real 


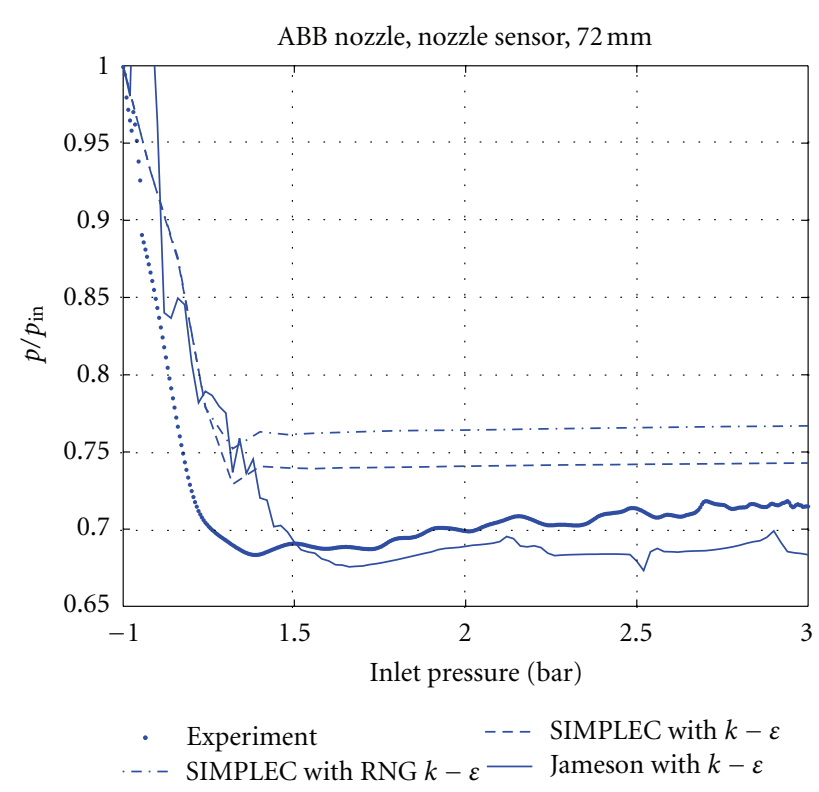

(a)

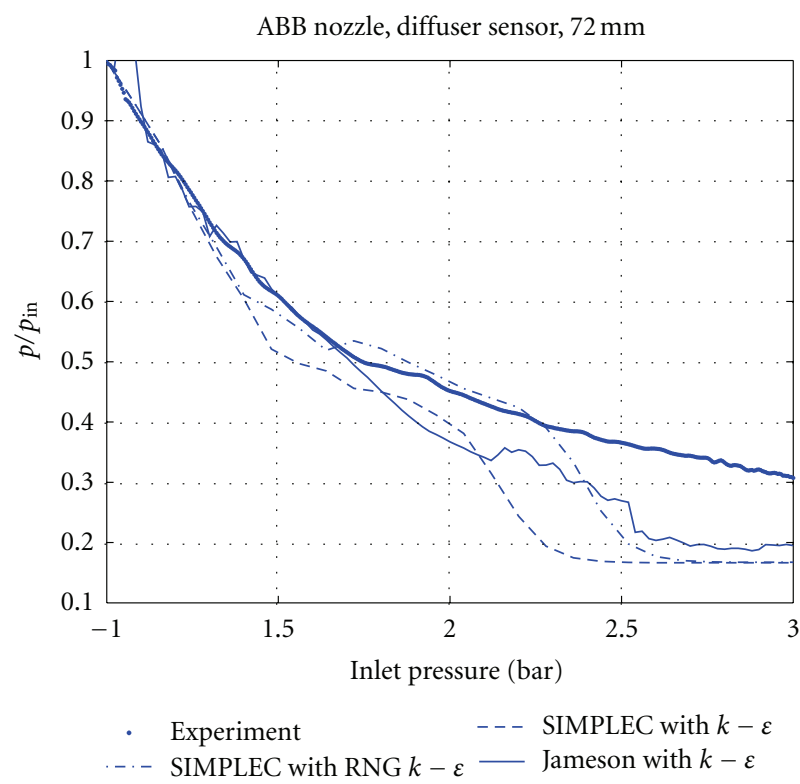

(b)

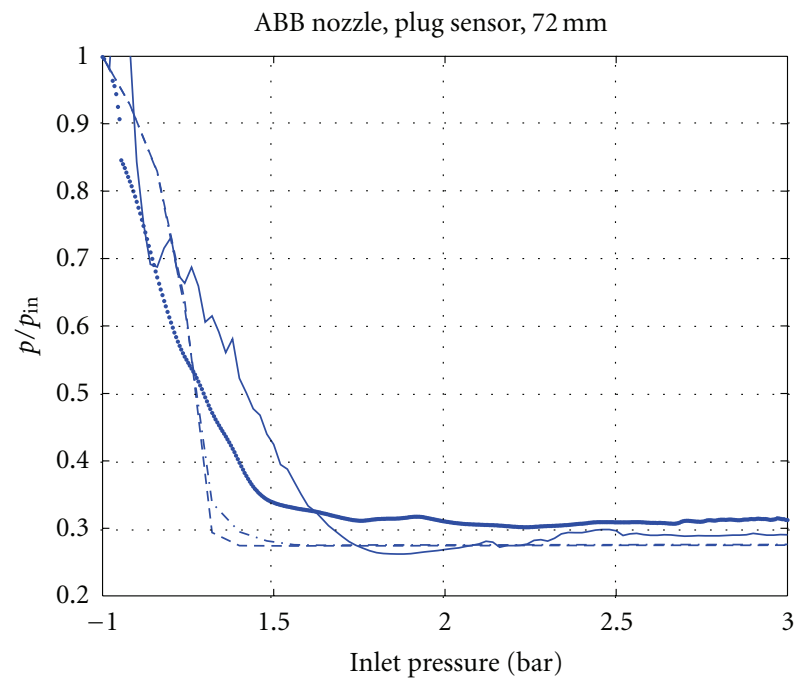

$\begin{array}{ll}\text { - } & \text { Experiment } \\ \cdots--- & \text { SIMPLEC with RNG } k-\varepsilon-\text { SIMPLEC with } k-\varepsilon \\ - & \text { Jameson with } k-\varepsilon\end{array}$

(c)

FIgURE 7: Comparison of the predicted pressure ratio and measurement for two different turbulence model.

breaker geometry as shown in Figure 8 is used. For the validation, a no-load switching test has been performed, and the transient pressure data at three different locations in the transonic diffuser area have been monitored.

Figure 8 shows the sketch of the main flow region and locations of pressure sensors of the tested high-voltage circuit breaker. The pressure build-up measurement at the heating chamber has been applied as the inflow boundary of the flow region. The initial filling pressure is set to be 6 bar. As the heating volume compresses, the inlet pressure reaches up to 12 bar, and the big difference between the inflow pressure and the filling pressure generates transonic flow in the diffuser area where the pressure sensors are implemented. Figure 9 shows the comparison of the simulated and the measured pressure data at different sensor locations. For the simulations, the second-order space accuracy and the firstorder time accuracy have been adopted for the SIMPLECbased solver. The time steps are chosen to be small enough to guarantee that the flow solver shows consistent transient behavior. In order to simulate the moving contacts, a deformable mesh has been used. Note that the simulated pressure curve at time zero does not start with the filling pressure, 6 bar. The reason for this is that the deformable mesh option in the used solver does not allow completely 


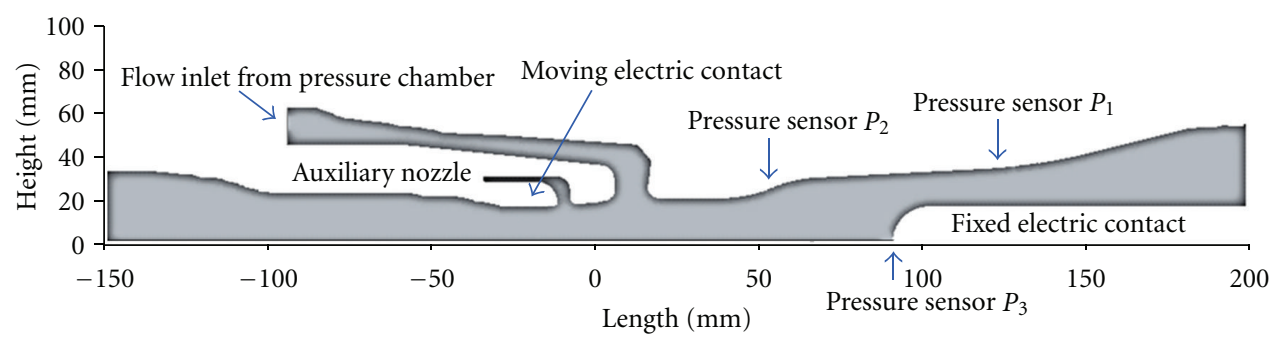

FIGURE 8: The illustrative sketch of the main flow region of the tested high-voltage circuit breaker and the locations of the three pressure sensors.

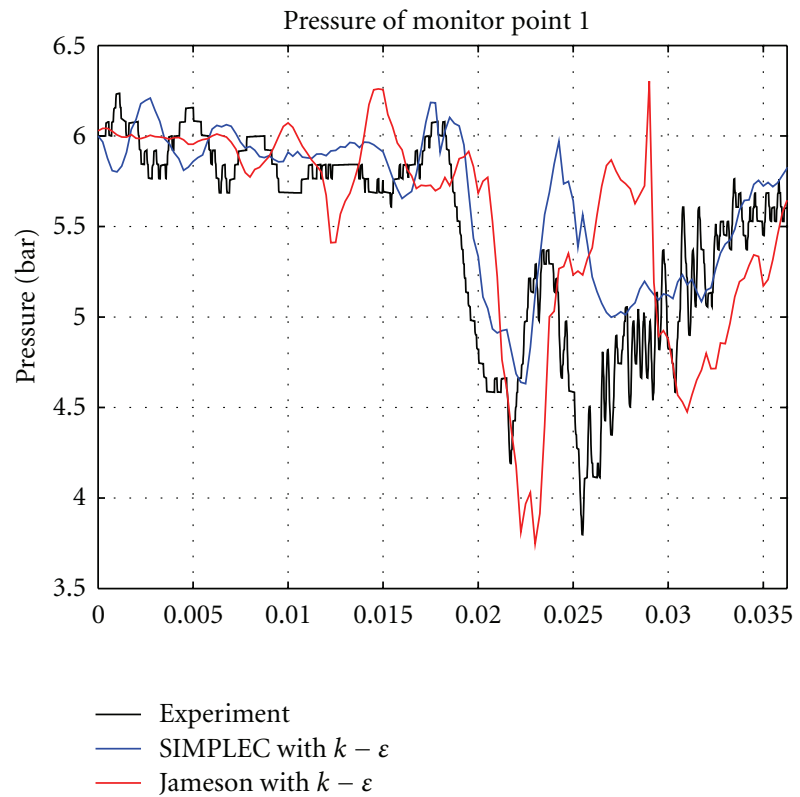

(a)

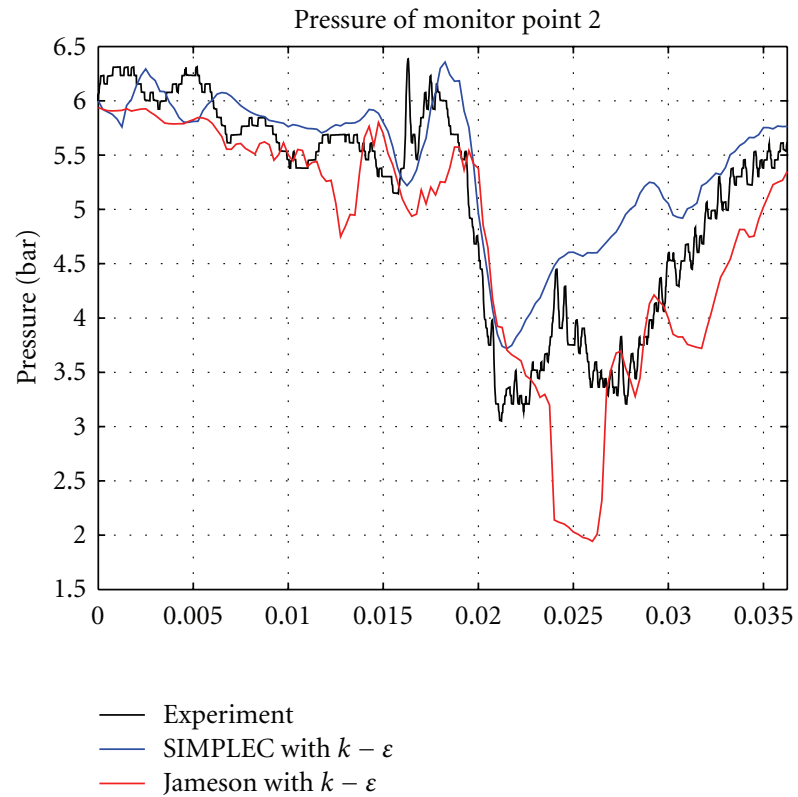

(b)

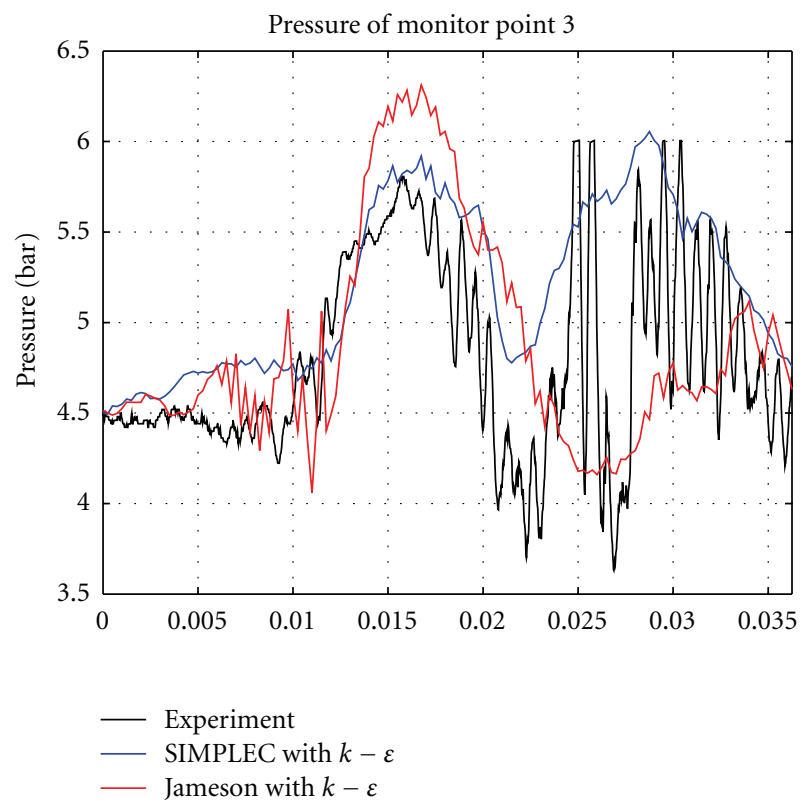

(c)

FIGURE 9: Comparison of measurement (black) with simulation with Jameson method (blue) and SIMPLEC (red) for three different monitor points. 


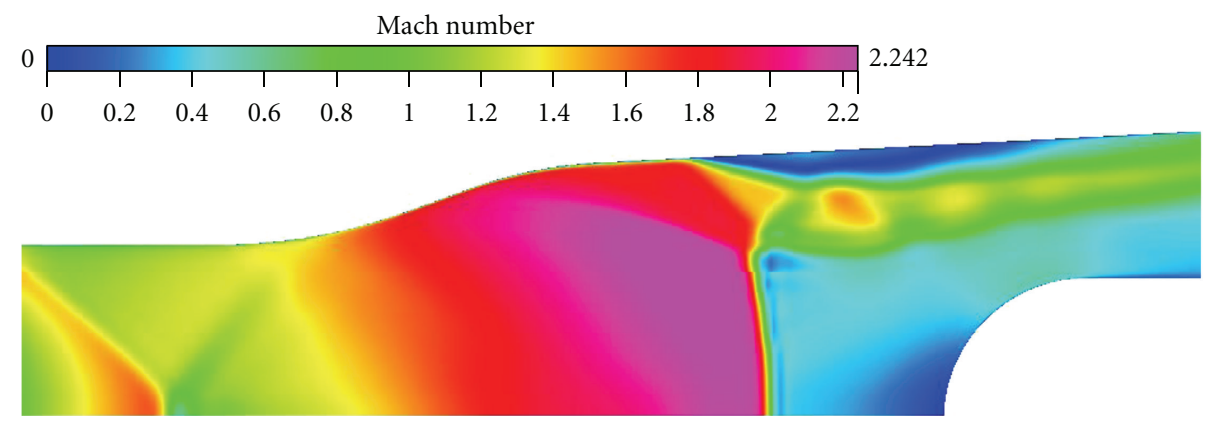

(a)

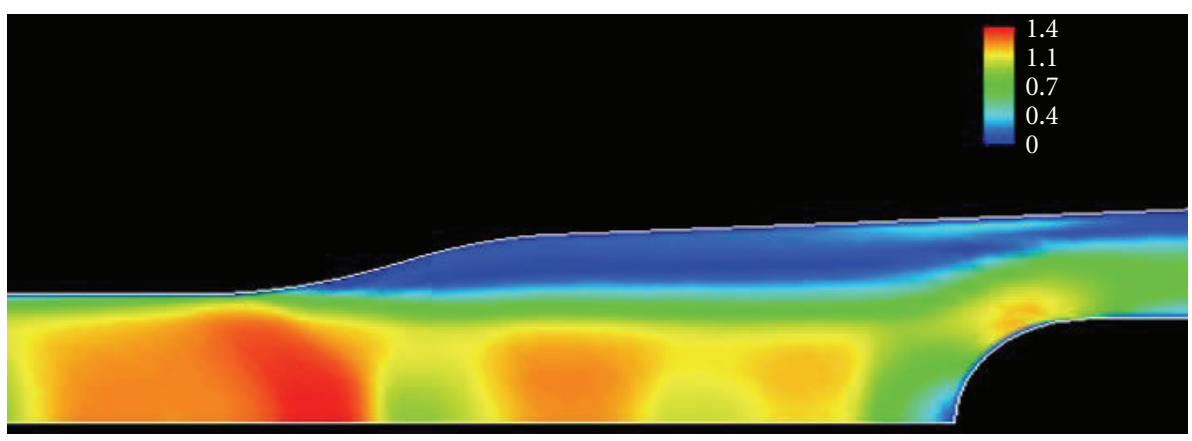

(b)

FIgURE 10: Comparison of simulated Mach number configurations at $t=24 \mathrm{~ms}$ with algorithm of (a) SIMPLEC (b) Jameson.

closed contact position. Therefore, the initial configuration of the transient simulation has been calculated by a steady simulation for a very small contact opening and a very small pressure build-up at the inlet.

For the CFD simulations with the algorithm based on Jameson's method, the explicit Range-Kutta time marching with the second-order accuracy has been used. The space resolution is the standard 2nd-order central difference. In order to stabilize the shock discontinuities, an artificial damping has been applied where necessary. For the moving contacts, CHIMERA mesh is used such that the transient simulation can start from the completely closed contact positions; see [5] for details of the simulation method.

Though not shown in Figure 9, we observed that the SIMPLEC-based simulation well predicts the pressure values in the region beginning from the pressure inlet up to the stagnation point on the symmetry axis, where subsonic flow features dominate. However, as it is shown in Figure 9, it fails to predict the transient pressure values at the monitoring points 1, 2, and 3, both in quantitative and qualitative sense. Note that compressible flow features dominate at these monitoring points. On the other hand, Figure 9 shows that the density-based explicit solver captures the qualitatively correct transient flow behavior, though it shows a deviation from the measured quantitative pressure value at each monitoring point. The reason why it captures the transient dynamics better than the semi-implicit SIMPLEC method is that it resolves the sonic wave which transports the flow information, by restricting time step sizes small enough to obey CFL constraints. The big difference between the two tested algorithms is manifested at time near $t=24 \mathrm{~ms}$. The simulated Mach number configurations at $t=24 \mathrm{~ms}$ are compared in Figure 10.

There is a qualitative difference in transonic nozzle flow features between the two solvers. The SIMPLEC-methodbased solver predicts the supersonic flow attached to the diffuser wall. As the diffuser cross-section area increases, the supersonic flow expands fully before it is terminated by the vertical shock in front of the plug tip. The sensors 1 and 2 are located in the upstream of the shock, where the pressure of the fully expanded supersonic flow is very much low. This is why the SIMPLEC-based solver underestimates the pressure at sensor points 1 and 2. The simulated supersonic flow predicted more expanded supersonic flow with much stronger vertical shock than real. The simulated overexpanded supersonic flow in the diffuser also has an influence on the calculated pressure at the stagnation point just in front of the plug. The SIMPLEC-based simulation underestimates the pressure value at sensor 3, because it suffers from the higher total pressure loss across the strong shock.

On the other hand, the Jameson-method-based solver predicts the supersonic flow which is already detached at the diffuser inlet. The detached jet flow forms a shock bottle system. Sensors 1 and 2 are definitely located at the downstream region after the vertical shock, where the flow is subsonic. The overexpanded subsonic flow then results in higher pressure. This is why the Jameson-method-based solver slightly over-estimates the pressure at sensor points 1 and 2 . 


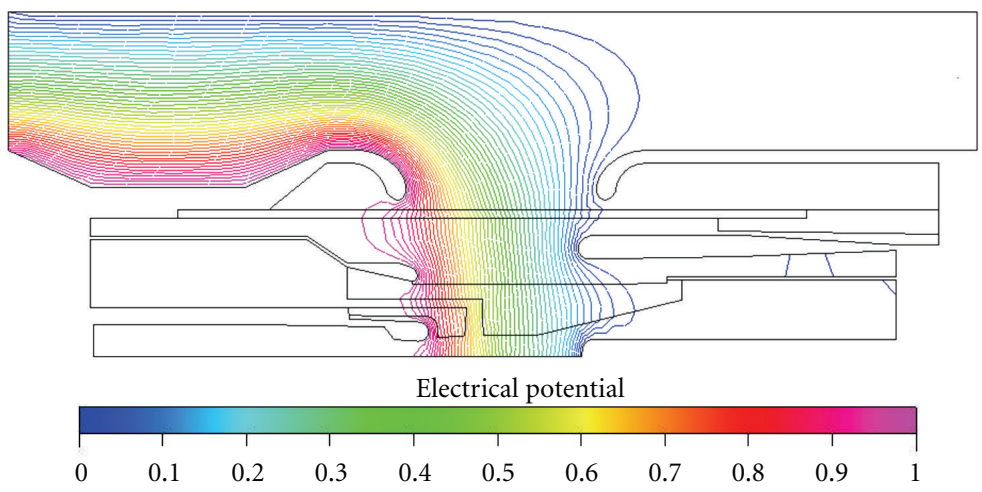

Figure 11: Typical electrostatic field distribution for a chosen contact separation.

To summarize, the Jameson-method-based CFD simulation shows better shock capturing capability than the SIMPLEC-method-based solver. The transient shock movement is also better captured by the Jameson-method-based explicit solver, since the semi-implicit SIMPLEC-based solver intrinsically introduces additional numerical viscosity.

\section{Influence of Solver Algorithm on Breakdown Voltage Prediction}

The prediction of breakdown voltages in capacitive switching tests of high-voltage circuit breaker is a challenging engineering issue. The breakdown voltage is estimated from the known dielectric breakdown criterion in $\mathrm{SF}_{6}$ gas, which is based on inputs from local flow and electrostatic field configurations. Particularly, the dielectric breakdown voltage $U_{\mathrm{bd}}$ is

$$
U_{\mathrm{bd}}=\left[C \frac{\rho}{\hat{E}}\right]_{\min } .
$$

Here, $\rho$ is the local flow density, and $\hat{E}$ is the local electric field strength when a unit voltage is applied to both contacts. The coefficient $C$ contains the information of influences from the surface roughness, area, and voltage frequency on breakdown voltage. The measurements from cold dielectric experiments determine the contents of $C$. From (2), for the reliable prediction of dielectric breakdown voltage, it is required to know local distribution of flow and electrostatic fields in the breaking chamber for given nozzle and diffuser geometry.

The electrostatic potential can be calculated by solving the following elliptic equation:

$$
\nabla \cdot(\varepsilon \nabla \phi)=0
$$

Here, $\varepsilon$ is the permittivity of the material, and the field strength $\vec{E}$ is calculated by $\vec{E}=-\nabla \phi$. Equation (2) can be solved either by a finite element or finite volume methods, with a high accuracy. Figure 11 shows typical equipotential lines of the electrical field E for a chosen contact separation. While the electric fields can be accurately computed with most of the commercial tools, the prediction of the flow fields poses a more difficult problem.
The breakdown voltages of two capacitive switching test duties of real high-voltage circuit breaker have been estimated. The local flow density is calculated by two different solver algorithms. The electrostatic field is calculated by a third electromagnetic simulation tool. The value of density to electrical field ratio in (2) is estimated by the superposition of the electric field and gas density field distribution as function of time. Figure 12 shows the comparison of the simulated breakdown voltages with the measurements. It can be observed that the CFD calculation with the SIMPLEC method underestimates the breakdown voltage by $10 \%$. This is due to the overestimation of the compressibility of the flow, which expands the supersonic flow at the diffuser area than in reality. This trend is amplified at nozzle opening. Note that the dielectrically most critical point in general is on the plug surface which is located in the diffuser region. On the other hand, the Jameson-method-based CFD tool shows smaller numerical uncertainty. This is mainly due to more precise capturing of the nonlinear flow structure, since the solver controls the conservation law better than the SIMPLEC method.

\section{Conclusions}

The conservative-density-based explicit flow solver algorithm is superior to SIMPLEC-pressure-correction-methodbased CFD solver, in predicting dielectric breakdown voltages in capacitive switching test duties of high-voltage circuit breakers with over-critical pressure ratio.

The SIMPLEC-based simulation shows higher numerical uncertainties than the density-based one, in predicting highly dynamic transonic flow configuration inside the circuit breaker diffuser. The main reason is that while the SIMPLEC scheme is optimal in simulating steady incompressible flow dynamics, it often fails to capture correct nonlinear wave structure of the compressible flow. However, the advantage of SIMPLEC method is that it allows larger computational time step with better numerical stability. But, it diffuses fine time scale transient dynamics away, even if the solution is well converged. 
Breakdown voltage comparison (based on semi-implicit CFD solver)

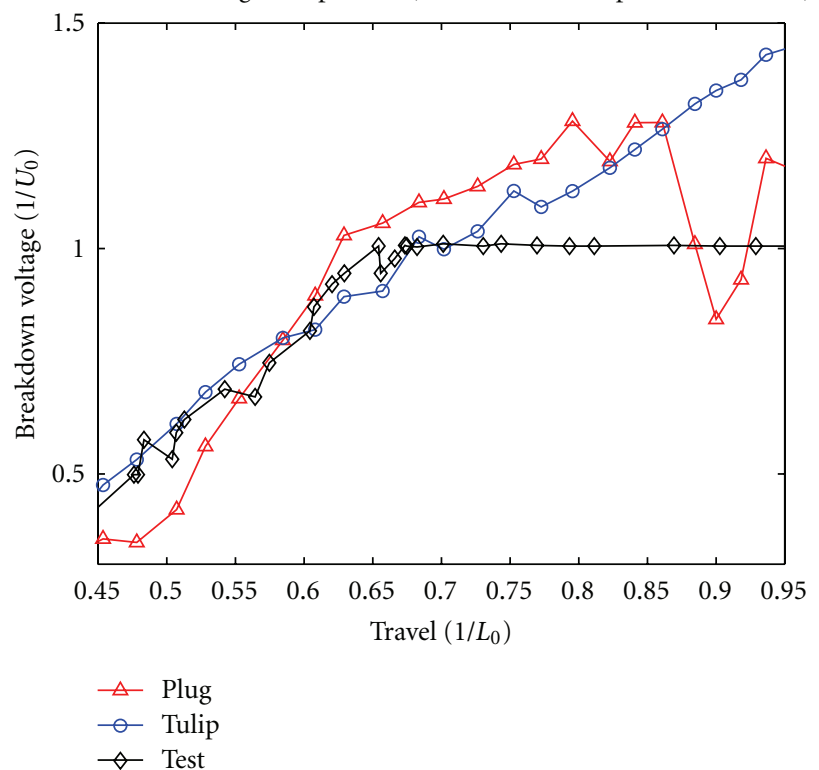

(a)

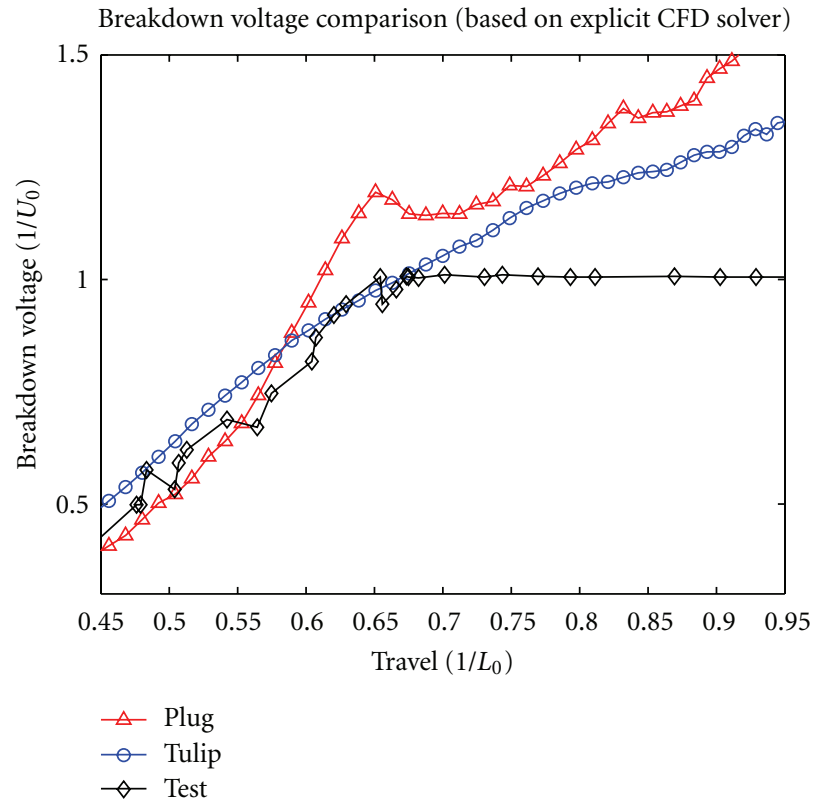

(b)

FIGURE 12: Comparison of the measured breakdown voltages with simulation with (a) SIMPLEC (b) Jameson algorithm.

There is significant influence of turbulence model. The shock-induced flow separation has been shown to be governed by the detailed modeling of the shock and the boundary layer interaction. The modeling issues should be further investigated and the optimal modeling concept should be accordingly identified.

The presented study shows that adopting optimal CFD algorithm for dynamic transonic flow simulation inside the breaking chamber of high-voltage circuit breaker can substantially improve the prediction power of dielectric breakdown voltage in capacitive switching of high-voltage circuit breaker. The conservative-density-based solver with advanced turbulence modeling such as RNG $k-\varepsilon$ is recommended.

\section{References}

[1] A. Jameson, W. Schmidt, and E. Turkel, "Numerical solutions of the Euler equations by finite volume methods using Rung-Kutta time-stepping schemes," in Proceedings of the 14th Fluid and Plasma Dynamic Conference, Palo Alto, Calif, USA, June 1981.

[2] J. P. Van Doormaal and G. D. Raithby, "Enhancements of the SIMPLE method for predicting incompressible fluid flows," Numerical Heat Transfer, vol. 7, no. 2, pp. 147-163, 1984.

[3] R. Courant, K. Friedrichs, and H. Lewy, "Über die partiellen Differenzengleichungen der mathematischen Physik," Mathematische Annalen, vol. 100, no. 1, pp. 32-74, 1928.

[4] M. Sajben, T. J. Bogar, and J. C. Kroutil, "Forced oscillation experiments in supercritical diffuser flows," AIAA Journal, vol. 22, no. 4, pp. 465-474, 1984.

[5] X. Ye, K. Muller, and J. Stechbarth, "Progress in the flow simulation of high voltage circuit breakers," Finite Volumes for Complex Applications II, 1998. 

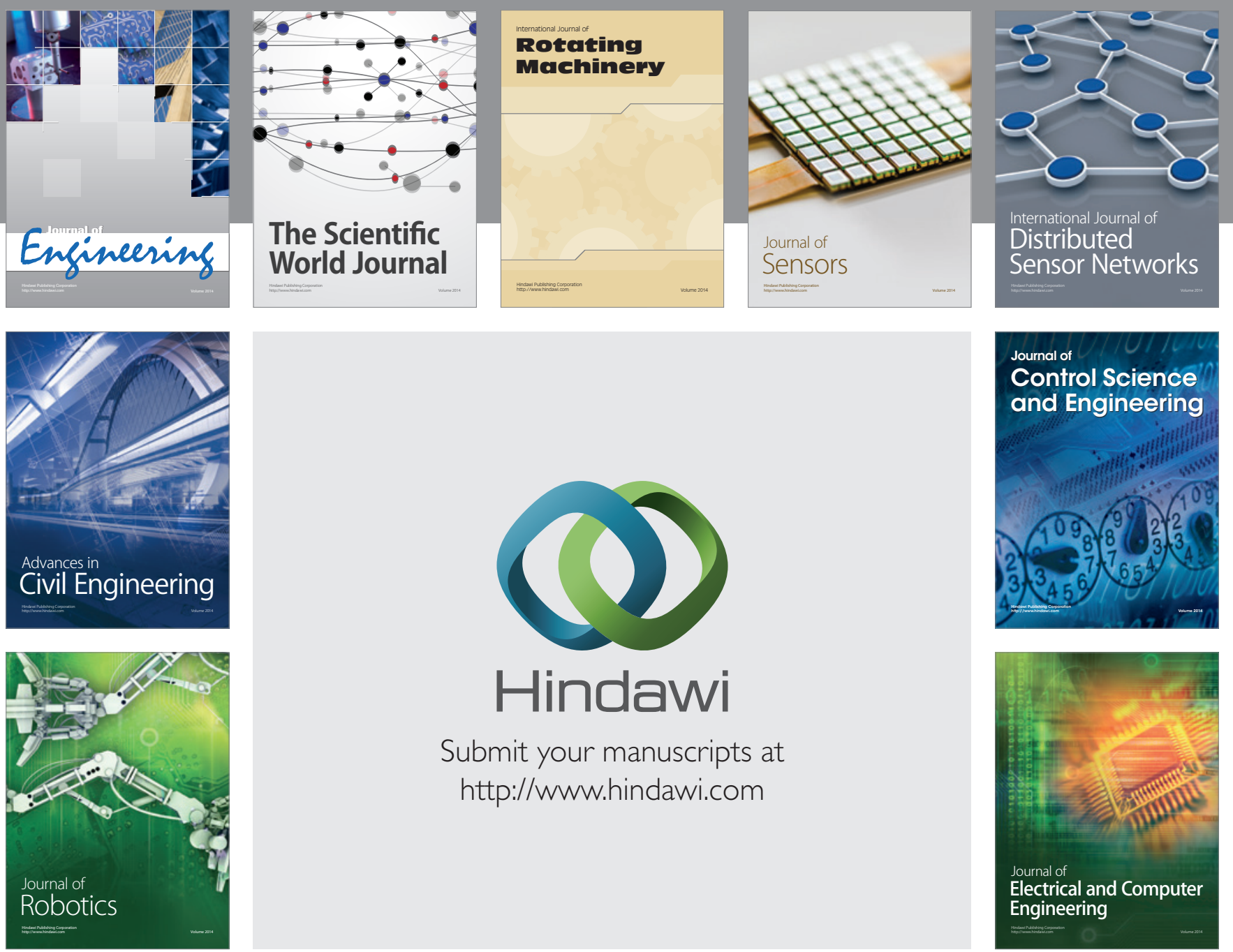

Submit your manuscripts at

http://www.hindawi.com
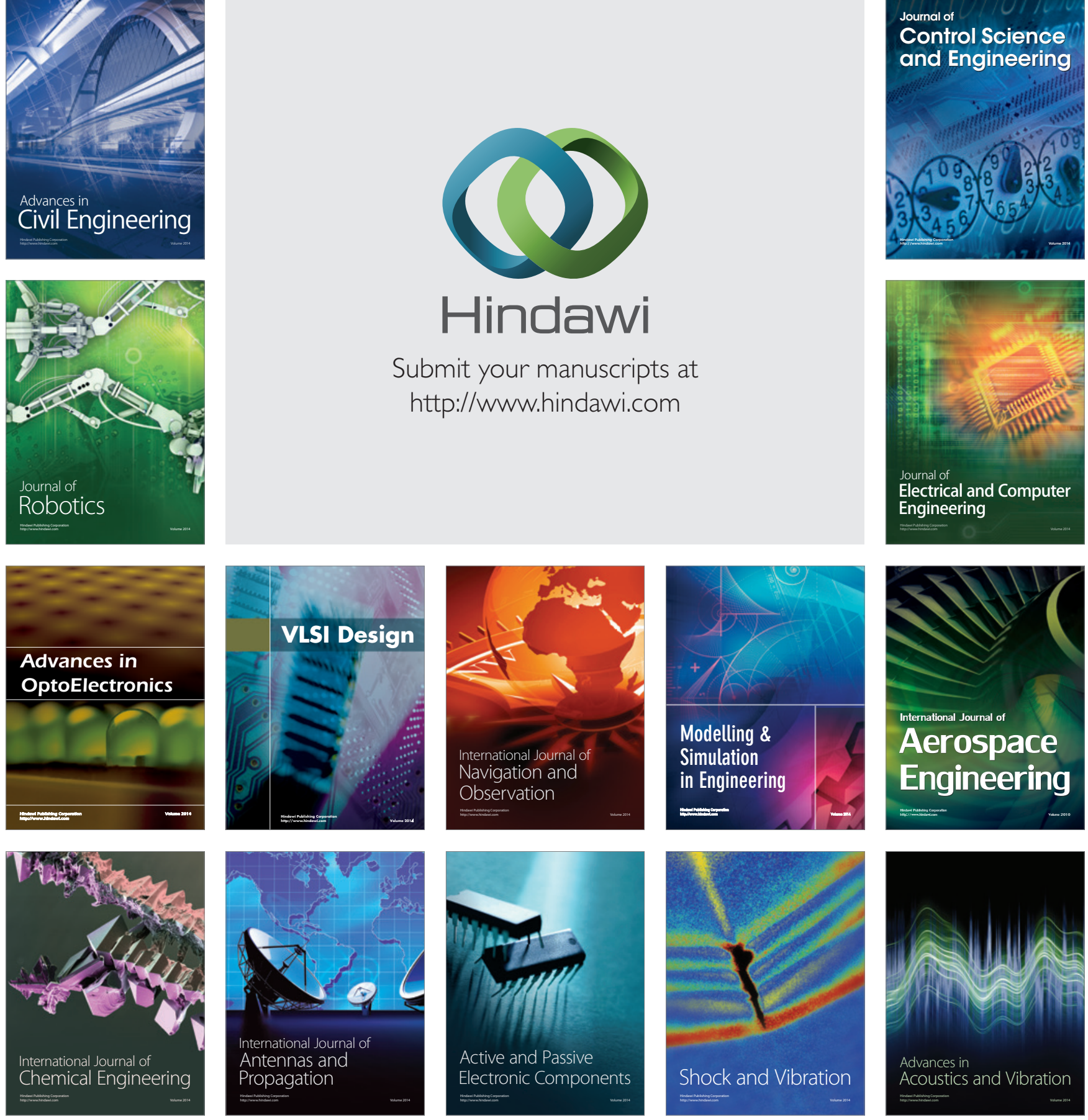